\title{
A New Approach to the Fine Kinney Method with AHP Based ELECTRE I and Math Model on Risk Assessment for Natural Disasters
}

\section{Doğal Afetler için AHP Tabanlı ELECTRE I ve Matematik Model ile Risk Değerlendirmesine Ilişkin Fine Kinney Yöntemine Yeni Bir Yaklaşım}

\section{Onur DERSE ${ }^{1}$ (D)}

${ }^{1}$ Lecturer, Tarsus University, Department of Management and Organization, Logistics, Mersin, Turkey

ORCID: O.D. 0000-0002-4528-1999

\section{ABSTRACT}

Natural disasters impose enormous risks on human living and the environment. Researchers have given more attention to evaluating these risks in the context of disasters. Studies focus on the risk assessment of only one of the natural disasters for the regions. However, a risk assessment should be conducted that includes all-natural disasters for these regions. This risk assessment is dealt with by 8 different provinces in the Aegean region of Turkey in the work. A case data (1990-2020) on natural disasters such as earthquake, fire, landslide, flood, storm/typhoon has been considered for the cities covered. By revising the Fine Kinney risk assessment method for natural disasters, a risk score is obtained for each province. Then, the AHP based ELECTRE I method is applied to these provinces. As a result of this method, the riskiest region is obtained. The location of a crisis center in the riskiest region obtained should ensure effective solutions to the regions which are affected by the results of the disasters. Thus, the problem of choosing the most suitable location in the crisis center is handled with a goal programming approach.

Keywords: Risk Assessment; Mathematical Modelling; Natural Disasters 


\section{INTRODUCTION}

Natural disasters are undoubtedly one of the most complicated events in the modern era. As the uncertainty involved increases, the variables need to be examined further (Li et al., 2013). Data on natural disasters were collected in different ways by various groups for different purposes (Dilley et al., 2005). Natural disasters have consisted of earthquakes, floods, landslides, fire, storm/typhoon, and many more, etc. Many papers have been conducted related to natural disasters in the world (Guo et al., 2017, Supriyadi et al., 2018, Ivčević et al., 2019, Eyre et al., 2020, Lee et al., 2020). Figure 1 depicts the distribution of several natural disasters in the world (Dilley et al., 2005).

Natural disasters are usually indistinguishable; which destroys everything in their way (Guidry and Margolis, 2005). However, assessment of the risks caused by natural disasters is known as the most important step to take precautions instead of preventing natural disasters. Luchuan (1999) and Xu et al., (2015) deals with risk assessment in a region for natural disasters. Emblemsvåg (2008) conducts risk analysis for a region in Norway for rock falls from natural disasters. Osipov et al. (2019) conduct a risk assessment for natural disasters such as earthquakes, floods and landslides.

In the field of risk assessment, the Fine Kinney method is widely used in practice (Kokangül et al., 2017). Kokangül et al. (2017) use the Fine Kinney method using a new approach to the classification of hazards in the health sector. Gul et al.
(2018) discuss the Fine Kinney method in the arms industry. Ersoy et al. (2019) use the Fine Kinney method for the excavation process that is observed in the marble quarry, possible accidents, and their determined effects. Y1lmaz and Özcan (2019) conducted a risk assessment and ranking integrating the AHP and the Fine Kinney method in their study. By this application, a different priority rank is created and which risk to be eliminated primarily was determined. The regional risk of natural disasters is a critical MCDM problem in the literature due to the complicated and usually conflicting evaluation index system (Chen et al., 2019). Yilmaz and Ozcan (2019) propose that the AHP method is one of the multi-criteria decision making (MCDM) methods used for risk. There are many different studies related to risk using the AHP method (Ganguly and Guin, 2013, Mabrouki et al., 2014; Dagsuyu et al., 2021). Also, ELECTRE, PROMETHEE, TOPSIS, VIKOR have been used for many years. Chen et al. (2019) use TOPSIS and VIKOR techniques to assess the risk of regions to natural hazards. The proposed approach not only ensures the listing of regions but also reveals the impact of indicators on regional risk. Sukcharoen et al. (2016) use GIS and MCDM practices to create a flood risk model in a region in Thailand. Being in the optimum position at the time of a natural disaster is very important for the quick access of the aid. For these reasons, various mathematical programming models are recommended for natural disasters. Hong and Jeong (2019) propose network design with a multi-purpose programming model for natural disasters. Ma et al. (2019) present site selection models for natural disaster shelters.
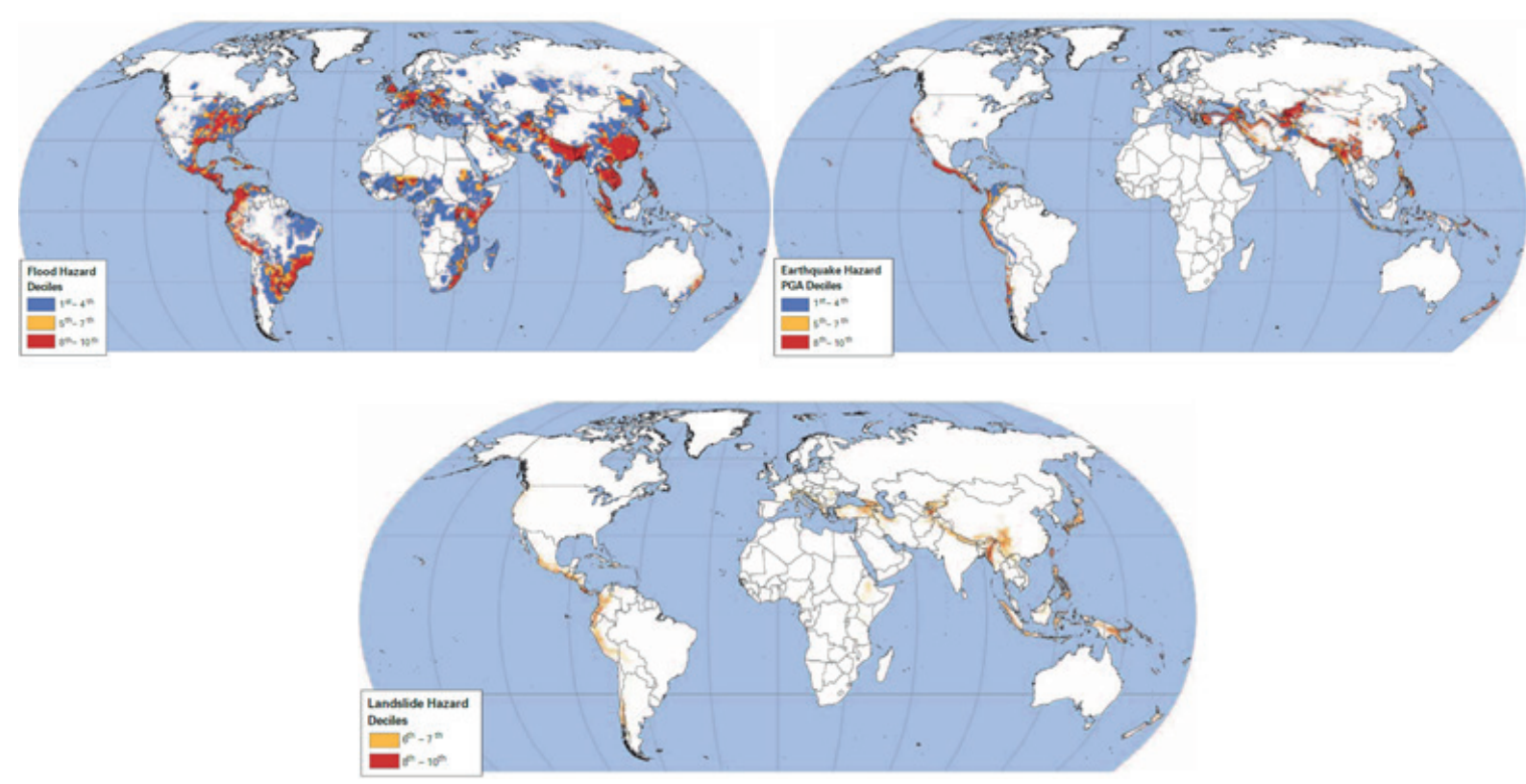

Figure 1: Distribution of Hazardous Areas by Hazard Type (Flood/Earthquake/Landslide) (Dilley et al., 2005). 
Natural disasters are generally evaluated on their own. However, it will be more accurate to determine the general risk value of the region by considering all-natural disasters together. The original contribution of the study is the consideration of natural disasters together for the region under consideration. In this study, the provincial natural disasters in Turkey's Aegean region (earthquakes, fires, landslides, storm/typhoons, landslides) are dealt with on a yearly basis. The Fine Kinney method, which is one of the risk analysis methods, was revised and used with a new approach for natural disasters. The revised Fine Kinney risk scores are obtained for all provinces. Among the risk scores obtained, to choose the riskiest region, and using different methods is needed since the risk score values were close. The ELECTRE I method, which is one of the MCDM methods, is used with the weights obtained from the AHP method. For the riskiest region, considering the set covering and population goals, the most suitable residential area is selected with the goal programming approach. In this study, other important original contributions are to bring a new approach to the Fine Kinney method and Besides, using different methods together.

\section{MATERIAL AND METHODS}

The present study develops a new approximation based on the Fine Kinney. Also, provinces are compared with an AHP based ELECTRE I approach. As a result, the selection of the most suitable region with the goal programming for the selected province is discussed.

\subsection{Fine Kinney method}

Kinney and Wiruth (1976) developed the Fine Kinney method. In this method, three parameters (probability, exposure, and possible results) are taken into consideration for each hazard detected and the risk score is obtained by multiplying these values. The parameters of probability, exposure, and possible results are depicted in Table 1, Table 2, and Table 3. Table 4 shows the risk score values (Kinney and Wiruth, 1976).

Table 1: Likelihood of Hazardous Event.

\begin{tabular}{lc}
\hline Probability & Value \\
\hline Might well be expected & 10 \\
Quite possible & 6 \\
Unusual but possible & 3 \\
Only remotely possible & 1 \\
Conceivable but very unlikely & 0.5 \\
Practically impossible & 0.2 \\
Virtually impossible & 0.1 \\
\hline
\end{tabular}

Table 2: The Exposure Factor.

\begin{tabular}{lc}
\hline Frequency & Value \\
\hline Continuous & 10 \\
Frequent (daily) & 6 \\
Occasional (weekly) & 3 \\
Unusual (monthly) & 2 \\
Rare (a few per year) & 1 \\
Very rare (yearly) & 0.5 \\
\hline
\end{tabular}

Table 3: Factors for Possible Consequences.

\begin{tabular}{lc}
\hline Severity & Value \\
\hline Catastrophe (many fatalities) & 100 \\
Disaster (few fatalities) & 40 \\
Very serious (fatality) & 15 \\
Serious (serious injury) & 7 \\
Important (disability) & 3 \\
Noticeable (minor first aid accident) & 1 \\
\hline
\end{tabular}

Table 4: Risk Score.

\begin{tabular}{ll}
\hline Fine Kinney Risk Score & Fine Kinney Risk Situation \\
\hline$>400$ & Very high risk; consider discontinuing operation \\
$200-400$ & High risk; immediate correction required \\
$70-200$ & Substantial risk; correction needed \\
$20-70$ & Possible risk; attention indicated \\
$>20$ & Risk; perhaps acceptable \\
\hline
\end{tabular}

\subsection{MCDM methods}

\subsubsection{AHP method}

AHP method, which was introduced in Saaty (1980), uses the comparison values in Table 5 below to compare the effects of different criteria on each other. The values in Table 5 will be used to weight the criteria.

Table 5: AHP comparison matrix.

\begin{tabular}{ll}
\hline Importance intensity & Definition \\
\hline 1 & Equal importance \\
3 & Moderate importance of one over another \\
5 & Strong importance of one over another \\
7 & Very strong importance of one over another \\
9 & The extreme importance of one over another \\
$2,4,6,8$ & Intermediate values \\
\hline
\end{tabular}

\subsubsection{ELECTRE I}

Pang et al. (2011) ELECTRE I steps in are applied.

Step 1 - Constructing the Decision Matrix

Step 2 - Construction of the Normalized Decision Matrix

Step 3 - Construction of the Weighted Normalized Decision Matrix

Step 4 - Determination of Concordance and Discordance Sets 
Step 5 - Calculation of Differentiation Measures

Step 6 - Superiority Comparison

Step 7 - Calculation of Net Concordance and Discordance Indices

As a result of these steps, there is the most suitable alternative.

\subsection{Mathematical Model}

The mathematical model can be divided into many subclasses. Goal programming is one of these classes. Goal programming is a mathematical model approach where multiple goals are used to achieve at the same time. The model developed for the region under consideration is expressed as follows.

$$
\begin{aligned}
& \text { Subject to } \\
& \sum_{j}^{J} w_{j} \cdot y_{j}=d_{1}^{+}-d_{1}^{-} \\
& \sum_{j}^{J} u_{j j} y_{j}=d_{2}^{+}-d_{2}^{-} \\
& \sum_{j}^{J} y_{j}=p \\
& \sum_{j}^{J} u_{j j} y_{j} \geq S C
\end{aligned}
$$$$
\text { Minimize } \mathrm{z}=d_{1}^{+}+d_{1}+d_{2}^{+}
$$

$$
\begin{aligned}
& d_{1}^{+}, d_{1}^{-}, d_{2}^{+}, d_{2}^{-}, \mathrm{p}, \mathrm{z} \geq 0 \\
& \mathrm{y}_{\mathrm{j}}=(0,1)
\end{aligned}
$$

The $\mathrm{j}$ index used in the equation represents the fields. Equation 1 shows the deviation values from the goals. Equation 2 is the constraint to select a region with a high population. Equation 3 is the constraint to minimize the distance of the selected region. Equation 4 shows the total number of selected regions. Equation 5 is the set covering constraint. Equation 6 shows positive variables. Equation 7 shows the binary variable.

\section{A NEW APPROACH FOR THE FINE KINNEY METHOD}

Risk assessment is used for natural disasters. One of the

\begin{tabular}{|c|c|c|c|c|c|c|}
\hline Probability & Value & $\begin{array}{l}\text { Revised Probability } \\
\text { for Fire }\end{array}$ & $\begin{array}{l}\text { Revised Probability } \\
\text { for Earthquake }\end{array}$ & $\begin{array}{l}\text { Revised Probability } \\
\text { for Landslide }\end{array}$ & $\begin{array}{l}\text { Revised Probability } \\
\text { for Storm / Typhoon }\end{array}$ & $\begin{array}{l}\text { Revised Probability } \\
\text { for Flood }\end{array}$ \\
\hline Might well be expected & 10 & $\begin{array}{l}\text { 1.level (the riskiest } \\
\text { region) }\end{array}$ & $\begin{array}{l}\text { 1.level (the riskiest } \\
\text { region) }\end{array}$ & $\begin{array}{l}\text { 1.level (the riskiest } \\
\text { region) }\end{array}$ & $\begin{array}{l}\text { 1.level (the riskiest } \\
\text { region) }\end{array}$ & $\begin{array}{l}\text { 1.level (the riskiest } \\
\text { region) }\end{array}$ \\
\hline Quite possible & 6 & $\begin{array}{l}\text { 2.level (potentially risky } \\
\text { area) }\end{array}$ & $\begin{array}{l}\text { 2.level (potentially risky } \\
\text { area) }\end{array}$ & $\begin{array}{l}\text { 2.level (potentially risky } \\
\text { area) }\end{array}$ & $\begin{array}{l}\text { 2.level (potentially risky } \\
\text { area) }\end{array}$ & $\begin{array}{l}\text { 2.level (potentially risky } \\
\text { area) }\end{array}$ \\
\hline Unusual but possible & 3 & $\begin{array}{l}\text { 3.level (unusual but } \\
\text { possible) }\end{array}$ & $\begin{array}{l}\text { 3.level (unusual but } \\
\text { possible) }\end{array}$ & $\begin{array}{l}\text { 3.level (unusual but } \\
\text { possible) }\end{array}$ & $\begin{array}{l}\text { 3.level (unusual but } \\
\text { possible) }\end{array}$ & $\begin{array}{l}\text { 3.level (unusual but } \\
\text { possible) }\end{array}$ \\
\hline Only remotely possible & 1 & $\begin{array}{l}\text { 4.level (only remotely } \\
\text { possible) }\end{array}$ & $\begin{array}{l}\text { 4.level (only remotely } \\
\text { possible) }\end{array}$ & $\begin{array}{l}\text { 4.level (only remotely } \\
\text { possible) }\end{array}$ & $\begin{array}{l}\text { 4.level (only remotely } \\
\text { possible) }\end{array}$ & $\begin{array}{l}\text { 4.level (only remotely } \\
\text { possible) }\end{array}$ \\
\hline $\begin{array}{l}\text { Conceivable but very } \\
\text { unlikely }\end{array}$ & 0.5 & $\begin{array}{l}5 . \text { level (Conceivable but } \\
\text { very unlikely) }\end{array}$ & $\begin{array}{l}\text { 5.level (Conceivable but } \\
\text { very unlikely) }\end{array}$ & $\begin{array}{l}\text { 5.level (Conceivable but } \\
\text { very unlikely) }\end{array}$ & $\begin{array}{l}\text { 5.level (Conceivable but } \\
\text { very unlikely) }\end{array}$ & $\begin{array}{l}\text { 5.level (Conceivable but } \\
\text { very unlikely) }\end{array}$ \\
\hline Practically impossible & 0.2 & $\begin{array}{l}\text { 6.level (practically } \\
\text { impossible) }\end{array}$ & $\begin{array}{l}\text { 6.level (practically } \\
\text { impossible) }\end{array}$ & $\begin{array}{l}\text { 6.level (practically } \\
\text { impossible) }\end{array}$ & $\begin{array}{l}\text { 6.level (practically } \\
\text { impossible) }\end{array}$ & $\begin{array}{l}\text { 6.level (practically } \\
\text { impossible) }\end{array}$ \\
\hline Virtually impossible & 0.1 & - & $\begin{array}{l}\text { 7.level (virtually } \\
\text { impossible) }\end{array}$ & - & - & - \\
\hline
\end{tabular}
methods used is the Fine Kinney risk assessment method. However, this method needs to be revised for natural disasters.

\begin{tabular}{|c|c|c|}
\hline Frequency & Value & Revised Frequency \\
\hline Continuous & 10 & A natural disaster that occurs approximately every month \\
\hline Frequent (daily) & 6 & A natural disaster that takes place approximately every two months \\
\hline Occasional (weekly) & 3 & A natural disaster that takes place approximately three or four times a year \\
\hline Unusual (monthly) & 2 & A natural disaster that occurs approximately twice a year \\
\hline Rare (a few per year) & 1 & A natural disaster that occurs approximately once a year \\
\hline Very rare (yearly) & 0.5 & Natural disaster less than once a year \\
\hline
\end{tabular}
The revised version of the Fine Kinney method developed by Kinney and Wiruth (1976) for natural disasters is as below in Table 6, Table 7, Table 8, and Table 9. Table 6 depicts the

Table 6: Revised Likelihood of Hazardous Event.

Table 7: Revised Exposure Factor.

Table 8: Revised Factors for Possible Consequences.

\begin{tabular}{lll}
\hline Severity & Value & Revised Severity \\
\hline Catastrophe (many fatalities) & 100 & Deaths \\
Disaster (few fatalities) & 40 & Death or Destroyed Buildings \\
Very serious (fatality) & 15 & Injured \\
Serious (serious injury) & 7 & Damaged Buildings \\
Important (disability) & 3 & - \\
Noticeable (minor first aid accident) & 1 & No damage / No injured \\
\hline
\end{tabular}

Table 9: Revised Risk Score.

\begin{tabular}{lll}
\hline $\begin{array}{l}\text { Fine Kinney Risk } \\
\text { Score }\end{array}$ & $\begin{array}{l}\text { Fine Kinney Risk } \\
\text { Situation }\end{array}$ & $\begin{array}{l}\text { Revised Fine Kinney } \\
\text { Risk Value }\end{array}$ \\
\hline$>400$ & Very high risk & 5 \\
$200-400$ & High risk & 4 \\
$70-200$ & Substantial risk & 3 \\
$20-70$ & Possible risk & 2 \\
$>20$ & Risk; perhaps acceptable & 1 \\
\hline
\end{tabular}


probability values revised to cover the range for each natural disaster occurred in the region.

In Table 7, the incident frequencies that occurred in natural disasters are compared with the frequency values of the Fine Kinney method and revised frequency values are obtained.

In Table 8, the severity occurred in the natural disasters are created by comparing the severity values of the Fine Kinney method.

Table 9 depicts the revised Fine Kinney Risk scores. While the Fine Kinney Risk Score is depicted in Equation 8, in the revised Fine Kinney Risk Score it is depicted in Equation 9.

Fine Kinney Risk Score $=$ Likelihood of Hazardous Event $\mathrm{x}$ Exposure Factor x Possible Consequences

If (Fine Kinney Risk Situation > 400)

Revised Fine Kinney Risk Value = 5;

else If(200 < Fine Kinney Risk Situation < 400)

Revised Fine Kinney Risk Value = 4;

else If(70 < Fine Kinney Risk Situation $<200)$

Revised Fine Kinney Risk Value = 3;

else If $(20<$ Fine Kinney Risk Situation $<70)$

Revised Fine Kinney Risk Value = 2;

else (Fine Kinney Risk Situation $<20$ )

Revised Fine Kinney Risk Value = 1;

\section{APPLICATIONS AND RESULTS}

\subsection{Application and Result for The New Fine Kinney Method}

This case is carried out in the Aegean region of Turkey. There are 8 provinces in the Aegean region. These provinces; Afyonkarahisar, Aydın, Denizli, İzmir, Kütahya, Manisa, Muğla, and Uşak are the provinces. The most common natural disasters in the Aegean region are taken into consideration. Fire, earthquake, landslide, storm/typhoon, and flood natural disasters are taken into consideration for each province. Frequency and Severity values are taken from the AFAD (Ministry of Interior Disaster and Emergency Management Presidency) page. Values are taken from 1990 to 2020. Probability values are taken by taking into consideration the regional risk level for each natural disaster. Table 10, Table 11, Table 12, Table 13, Table 14, Table 15, Table 16, Table 17 show the values of the Fine Kinney and the revised Fine Kinney for each province.

\subsection{Application and result for AHP based ELECTRE I}

By the results depicted in the tables, the risk scores of some provinces are higher. However, a different method is recommended for the provinces with high scores since the difference between the risk scores is not high. AHP based ELECTRE I method is recommended for Muğla, Manisa, Denizli, and İzmir provinces with risk scores of 13, 14, 15, and 15. Studies are using the ELECTRE I method based on AHP

Table 10: Fine Kinney and Revised Fine Kinney Scores for Afyonkarahisar.

\begin{tabular}{|c|c|c|c|c|c|c|c|c|c|c|}
\hline Hazards & $\begin{array}{l}\text { Revised } \\
\text { Probability for } \\
\text { Natural Disasters }\end{array}$ & $\begin{array}{l}\text { Revised } \\
\text { Frequency }\end{array}$ & $\begin{array}{l}\text { Revised } \\
\text { Frequency }\end{array}$ & Deaths & $\begin{array}{l}\text { Death or } \\
\text { Destroyed } \\
\text { Buildings }\end{array}$ & Injured & $\begin{array}{l}\text { Damaged } \\
\text { Buildings }\end{array}$ & $\begin{array}{l}\text { Revised } \\
\text { Severity }\end{array}$ & $\begin{array}{l}\text { Fine Kinney } \\
\text { Risk Score }\end{array}$ & $\begin{array}{l}\text { Revised Fine } \\
\text { Kinney Risk } \\
\text { Value }\end{array}$ \\
\hline Fire & 10 & 11 & 0.5 & & & $6 * 15$ & $4 * 7$ & $\begin{array}{l}(90+28) / 10 \\
=11.8\end{array}$ & 59 & 2 \\
\hline Earthquake & 6 & 46 & 2 & $8 * 100$ & $20 * 40$ & $10 * 15$ & $59269 * 7$ & 7.03 & 84.36 & 3 \\
\hline Landslide & 0.2 & 7 & 0.5 & & & & $7 * 7$ & 7 & 0.7 & 1 \\
\hline Storm/Typhoon & 1 & 22 & 1 & & & & $1 * 7$ & 7 & 7 & 1 \\
\hline Flood & 0.5 & 32 & 1 & & & & $49 * 7$ & 7 & 3.5 & 1 \\
\hline TOTAL Risk Score & & & & & & & & & 154.56 & 8 \\
\hline
\end{tabular}

Table 11: Fine Kinney and Revised Fine Kinney Scores for Aydın.

\begin{tabular}{|c|c|c|c|c|c|c|c|c|c|c|}
\hline Hazards & $\begin{array}{l}\text { Revised } \\
\text { Probability for } \\
\text { Natural Disasters }\end{array}$ & $\begin{array}{l}\text { Revised } \\
\text { Frequency }\end{array}$ & $\begin{array}{l}\text { Revised } \\
\text { Frequency }\end{array}$ & Deaths & $\begin{array}{l}\text { Death or } \\
\text { Destroyed } \\
\text { Buildings }\end{array}$ & Injured & $\begin{array}{l}\text { Damaged } \\
\text { Buildings }\end{array}$ & $\begin{array}{l}\text { Revised } \\
\text { Severity }\end{array}$ & $\begin{array}{l}\text { Fine Kinney } \\
\text { Risk Score }\end{array}$ & $\begin{array}{l}\text { Revised Fine } \\
\text { Kinney Risk } \\
\text { Value }\end{array}$ \\
\hline Fire & 10 & 127 & 4 & & & $4 * 15$ & $8 * 7$ & \multicolumn{2}{|c|}{$\begin{array}{l}(60+56) / 12=386.8 \\
9.67\end{array}$} & 4 \\
\hline Earthquake & 10 & 33 & 1 & & & & & 1 & 10 & 1 \\
\hline Landslide & 0.2 & 45 & 2 & & $5 * 40$ & & & 40 & 16 & 1 \\
\hline Storm/Typhoon & 3 & 23 & 1 & & $1 * 40$ & $2 * 15$ & & 23.33 & 69.99 & 2 \\
\hline Flood & 0.5 & 16 & 0.5 & & $6 * 40$ & & $120 * 7$ & 8.57 & 2.1425 & 1 \\
\hline TOTAL Risk Score & & & & & & & & & 484.9325 & 9 \\
\hline
\end{tabular}


Table 12: Fine Kinney and Revised Fine Kinney Scores for Denizli.

\begin{tabular}{|c|c|c|c|c|c|c|c|c|c|c|}
\hline Hazards & $\begin{array}{l}\text { Revised } \\
\text { Probability for } \\
\text { Natural Disasters }\end{array}$ & $\begin{array}{l}\text { Revised } \\
\text { Frequency }\end{array}$ & $\begin{array}{l}\text { Revised } \\
\text { Frequency }\end{array}$ & Deaths & $\begin{array}{l}\text { Death or } \\
\text { Destroyed } \\
\text { Buildings }\end{array}$ & Injured & $\begin{array}{l}\text { Damaged } \\
\text { Buildings }\end{array}$ & $\begin{array}{l}\text { Revised } \\
\text { Severity }\end{array}$ & $\begin{array}{l}\text { Fine Kinney } \\
\text { Risk Score }\end{array}$ & $\begin{array}{l}\text { Revised } \\
\text { Fine Kinney } \\
\text { Risk Value }\end{array}$ \\
\hline Fire & 6 & 56 & 2 & $4 * 100$ & $1 * 40$ & $1 * 15$ & $8 * 7$ & $\begin{array}{l}(400+40+15+56) \\
/ 14=36.5\end{array}$ & 438 & 5 \\
\hline Earthquake & 10 & 52 & 2 & & $100 * 40$ & & $100 * 7$ & 23.5 & 470 & 5 \\
\hline Landslide & 0.2 & 25 & 1 & & & & $566 * 7$ & 7 & 1.4 & 1 \\
\hline Storm/Typhoon & 1 & 28 & 1 & $6^{*} 100$ & & $3 * 15$ & & 71.67 & 71.67 & 3 \\
\hline Flood & 0.2 & 32 & 1 & $4 * 100$ & & $7 * 15$ & $496 * 7$ & 7.84 & 1.568 & 1 \\
\hline TOTAL Risk Score & & & & & & & & & 982.638 & 15 \\
\hline
\end{tabular}

Table 13: Fine Kinney and Revised Fine Kinney Scores for İzmir.

\begin{tabular}{|c|c|c|c|c|c|c|c|c|c|c|}
\hline Hazards & $\begin{array}{l}\text { Revised } \\
\text { Probability for } \\
\text { Natural Disasters }\end{array}$ & $\begin{array}{l}\text { Revised } \\
\text { Frequency }\end{array}$ & $\begin{array}{l}\text { Revised } \\
\text { Frequency }\end{array}$ & Deaths & $\begin{array}{l}\text { Death or } \\
\text { Destroyed } \\
\text { Buildings }\end{array}$ & Injured & $\begin{array}{l}\text { Damaged } \\
\text { Buildings }\end{array}$ & $\begin{array}{l}\text { Revised } \\
\text { Severity }\end{array}$ & $\begin{array}{l}\text { Fine Kinney } \\
\text { Risk Score }\end{array}$ & $\begin{array}{l}\text { Revised } \\
\text { Fine Kinney } \\
\text { Risk Value }\end{array}$ \\
\hline Fire & 10 & 194 & 6 & $4 * 100$ & & $6 * 15$ & $27 * 7$ & $\begin{array}{l}(400+90+189) \\
/ 37=18.35\end{array}$ & 1101 & 5 \\
\hline Earthquake & 10 & 115 & 3 & & & & $2141 * 7$ & 7 & 210 & 4 \\
\hline Landslide & 0.2 & 36 & 1 & & $2 * 40$ & $1 * 15$ & $32 * 7$ & 9.11 & 1.822 & 1 \\
\hline Storm/Typhoon & 3 & 59 & 2 & $6 * 100$ & & $10 * 15$ & $3 * 7$ & 40.58 & 243.48 & 4 \\
\hline Flood & 0.5 & 21 & 1 & & & $4^{*} 15$ & $101 * 7$ & 7.3 & 3.65 & 1 \\
\hline TOTAL Risk Score & & & & & & & & & 1559.952 & 15 \\
\hline
\end{tabular}

Table 14: Fine Kinney and Revised Fine Kinney Scores for Kütahya.

\begin{tabular}{|c|c|c|c|c|c|c|c|c|c|c|}
\hline Hazards & $\begin{array}{l}\text { Revised } \\
\text { Probability for } \\
\text { Natural Disasters }\end{array}$ & $\begin{array}{l}\text { Revised } \\
\text { Frequency }\end{array}$ & $\begin{array}{l}\text { Revised } \\
\text { Frequency }\end{array}$ & Deaths & $\begin{array}{l}\text { Death or } \\
\text { Destroyed } \\
\text { Buildings }\end{array}$ & Injured & $\begin{array}{l}\text { Damaged } \\
\text { Buildings }\end{array}$ & $\begin{array}{l}\text { Revised } \\
\text { Severity }\end{array}$ & $\begin{array}{l}\text { Fine Kinney } \\
\text { Risk Score }\end{array}$ & $\begin{array}{l}\text { Revised } \\
\text { Fine Kinney } \\
\text { Risk Value }\end{array}$ \\
\hline Fire & 6 & 60 & 2 & & $1 * 40$ & $2 * 15$ & $24 * 7$ & $\begin{array}{l}(40+30+168) / \\
27=8.81\end{array}$ & 105.72 & 3 \\
\hline Earthquake & 6 & 85 & 3 & $2 * 100$ & & $50 * 15$ & $1 * 7$ & 18.06 & 325.06 & 4 \\
\hline Landslide & 0.1 & 17 & 1 & & & & & 1 & 0.1 & 1 \\
\hline Storm/Typhoon & 1 & 21 & 1 & $6 * 100$ & & $6^{*} 15$ & & 57.5 & 57.5 & 2 \\
\hline Flood & 0.5 & 6 & 0.5 & & & & $50 * 7$ & 7 & 1.75 & 1 \\
\hline TOTAL Risk Score & & & & & & & & & 490.13 & 11 \\
\hline
\end{tabular}

Table 15: Fine Kinney and Revised Fine Kinney Scores for Manisa.

\begin{tabular}{|c|c|c|c|c|c|c|c|c|c|c|}
\hline Hazards & $\begin{array}{l}\text { Revised } \\
\text { Probability for } \\
\text { Natural Disasters }\end{array}$ & $\begin{array}{l}\text { Revised } \\
\text { Frequency }\end{array}$ & $\begin{array}{l}\text { Revised } \\
\text { Frequency }\end{array}$ & Deaths & $\begin{array}{l}\text { Death or } \\
\text { Destroyed } \\
\text { Buildings }\end{array}$ & Injured & $\begin{array}{l}\text { Damaged } \\
\text { Buildings }\end{array}$ & $\begin{array}{l}\text { Revised } \\
\text { Severity }\end{array}$ & $\begin{array}{l}\text { Fine Kinney } \\
\text { Risk Score }\end{array}$ & $\begin{array}{l}\text { Revised } \\
\text { Fine Kinney } \\
\text { Risk Value }\end{array}$ \\
\hline Fire & 10 & 82 & 3 & & $1 * 40$ & $2 * 15$ & $9 * 7$ & $\begin{array}{l}(40+30+63) \\
/ 12=11.08\end{array}$ & 332.4 & 4 \\
\hline Earthquake & 10 & 23 & 1 & & $1 * 40$ & & & 40 & 400 & 5 \\
\hline Landslide & 0.2 & 41 & 1 & & & $2 * 15$ & $29 * 7$ & 7.52 & 1.504 & 1 \\
\hline Storm/Typhoon & 3 & 37 & 1 & $11 * 100$ & & $7 * 15$ & $1 * 7$ & 63.79 & 191.37 & 3 \\
\hline Flood & 0.2 & 17 & 1 & $6 * 100$ & & & $430 * 7$ & 8.28 & 1.656 & 1 \\
\hline TOTAL Risk Score & & & & & & & & & 926.93 & 14 \\
\hline
\end{tabular}

Table 16: Fine Kinney and Revised Fine Kinney Scores for Muğla.

\begin{tabular}{|c|c|c|c|c|c|c|c|c|c|c|}
\hline Hazards & $\begin{array}{l}\text { Revised } \\
\text { Probability for } \\
\text { Natural Disasters }\end{array}$ & $\begin{array}{l}\text { Revised } \\
\text { Frequency }\end{array}$ & $\begin{array}{l}\text { Revised } \\
\text { Frequency }\end{array}$ & Deaths & $\begin{array}{l}\text { Death or } \\
\text { Destroyed } \\
\text { Buildings }\end{array}$ & Injured & $\begin{array}{l}\text { Damaged } \\
\text { Buildings }\end{array}$ & $\begin{array}{l}\text { Revised } \\
\text { Severity }\end{array}$ & $\begin{array}{l}\text { Fine Kinney } \\
\text { Risk Score }\end{array}$ & $\begin{array}{l}\text { Revised Fine } \\
\text { Kinney Risk } \\
\text { Value }\end{array}$ \\
\hline Fire & 10 & 384 & 10 & $7 * 100$ & & $16 * 15$ & $10 * 7$ & \multicolumn{2}{|c|}{$\begin{array}{l}(700+240+70) / 3061 \\
33=30.61\end{array}$} & 5 \\
\hline Earthquake & 10 & 62 & 2 & & & $9 * 15$ & $19 * 7$ & 9.57 & 191.4 & 3 \\
\hline Landslide & 0.2 & 18 & 1 & & & & $9 * 7$ & 7 & 1.4 & 1 \\
\hline Storm/Typhoon & 1 & 70 & 2 & $5 * 100$ & & $1 * 15$ & $1 * 7$ & 74.57 & 149.14 & 3 \\
\hline Flood & 0.5 & 19 & 1 & & & & $28 * 7$ & 7 & 3.5 & 1 \\
\hline TOTAL Risk Score & & & & & & & & & 3406.44 & 13 \\
\hline
\end{tabular}


Table 17: Fine Kinney and Revised Fine Kinney Scores for Uşak.

\begin{tabular}{|c|c|c|c|c|c|c|c|c|c|c|}
\hline Hazards & $\begin{array}{l}\text { Revised } \\
\text { Probability for } \\
\text { Natural Disasters }\end{array}$ & $\begin{array}{l}\text { Revised } \\
\text { Frequency }\end{array}$ & $\begin{array}{l}\text { Revised } \\
\text { Frequency }\end{array}$ & Deaths & $\begin{array}{l}\text { Death or } \\
\text { Destroyed } \\
\text { Buildings }\end{array}$ & Injured & $\begin{array}{l}\text { Damaged } \\
\text { Buildings }\end{array}$ & $\begin{array}{l}\text { Revised } \\
\text { Severity }\end{array}$ & $\begin{array}{l}\text { Fine Kinney } \\
\text { Risk Score }\end{array}$ & $\begin{array}{l}\text { Revised Fine } \\
\text { Kinney Risk } \\
\text { Value }\end{array}$ \\
\hline Fire & 10 & 21 & 1 & & & & $11 * 7$ & $77 / 11=7$ & 70 & 3 \\
\hline Earthquake & 6 & 0 & 0 & & & & & 1 & 0 & 1 \\
\hline Landslide & 0.1 & 9 & 0.5 & & & & & 1 & 0.05 & 1 \\
\hline Storm/Typhoon & 1 & 5 & 0.5 & & $1 * 40$ & $6 * 15$ & & 18.57 & 9.285 & 1 \\
\hline Flood & 0.2 & 2 & 0.5 & & & & $2200 * 7$ & 7 & 0.7 & 1 \\
\hline TOTAL Risk Score & & & & & & & & & 80.035 & 7 \\
\hline
\end{tabular}

(Pang et al., 2011). There are also many studies using the ELECTRE I method (Almeida, 2005, Hatami-Marbini and Tavana, 2011). In this method, Muğla, Manisa, Denizli, and İzmir provinces are used as an alternative. The criteria are the Fine Kinney Risk Scores, the revised Fine Kinney Risk Scores, and provincial populations. The reason why the population is taken as a criterion is that the population is too high to be affected in the event of natural disasters.

Table 18 shows the weighting of criteria with AHP.

After the AHP weights are obtained, ELECTRE I steps are applied.

Step 1 - Constructing the Decision Matrix

In Table 19, a decision matrix is formed for alternatives and criteria. Alternatives represent the provinces in the revised Fine Kinney method. Criteria represent total the Fine Kinney risk score, the total revised Fine Kinney Risk Score, and population amount. The values in the total Fine Kinney Risk score are evaluated to take into account the difference between the values in the same situation.

Step 2 - Construction of the Normalized Decision Matrix

Table 20 depicts the normalization of the decision matrix.

Step 3 -Construction of the Weighted Normalized Decision Matrix

The Weighted Normalized Decision Matrix in Table 21 is formed by multiplying the criteria weights obtained by the AHP method with the normalized decision matrix obtained in Table 20.

Table 18: AHP Weight calculation.

\begin{tabular}{|c|c|c|c|c|}
\hline The first part (scoring) & K1 (Fine Kinney Risk Score) & K2 (Revised Fine Kinney Risk Value) & K3 (Population amount) & Weight \\
\hline K1 (Fine Kinney Risk Score) & 1 & 1 & $3 / 1$ & 0,43 \\
\hline K2 (Revised Fine Kinney Risk Value) & 1 & 1 & $3 / 1$ & 0,43 \\
\hline K3 (Population amount) & $1 / 3$ & $1 / 3$ & 1 & 0,14 \\
\hline
\end{tabular}

Table 19: Application of Step 1.

\begin{tabular}{llll}
\hline & K1 (Fine Kinney Risk Score) & K2 (Revised Fine Kinney Risk Value) & K3 (Population amount) \\
\hline A1 (Denizli) & 982.638 & 15 & 1037208 \\
A2 (İmir) & 1559.952 & 15 & 4367251 \\
A3 (Manisa) & 926.93 & 14 & 1440611 \\
A4 (Muğla) & 3406.44 & 13 & 983142 \\
\hline
\end{tabular}

Table 20: Application of Step 2.

\begin{tabular}{|c|c|c|c|}
\hline & K1 (Fine Kinney Risk Score) & K2 (Revised Fine Kinney Risk Value) & K3 (Population amount) \\
\hline A1 (Denizli) & 0.25 & 0.53 & 0.22 \\
\hline A2 (İzmir) & 0.39 & 0.53 & 0.91 \\
\hline A3 (Manisa) & 0.23 & 0.49 & 0.3 \\
\hline A4 (Muğla) & 0.86 & 0.46 & 0.2 \\
\hline
\end{tabular}

Table 21: Application of Step 3.

\begin{tabular}{llll}
\hline & K1 (Fine Kinney Risk Score) & K2 (Revised Fine Kinney Risk Value) & K3 (Population amount) \\
\hline A1 (Denizli) & 0.1075 & 0.2279 & 0.0308 \\
A2 (İzmir) & 0.1677 & 0.53 & 0.1274 \\
A3 (Manisa) & 0.23 & 0.53 & 0.042 \\
A4 (Muğla) & 0.0989 & 0.1978 & 0.28 \\
\hline
\end{tabular}


Step 4 / Step 5-Determination of Concordance and Discordance Sets / Calculation of Differentiation Measures

The matrix $\mathrm{V}$ is used to determine the sets of concordance (C) and discordance (D).

\section{Concordance Sets}

In this set, two alternatives are compared and high-value ones are chosen. Table 22 is obtained by applying the necessary procedures.

Table 22: Concordance Index Values.

\begin{tabular}{lllll}
\hline & A1 (Denizli) & A2 (İzmir) & A3 (Manisa) & A4 (Muğla) \\
\hline A1 (Denizli) & - & 0 & 0 & 0.86 \\
A2 (ízmir) & 1 & - & 0.57 & 0.86 \\
A3 (Manisa) & 1 & 1 & - & 0.86 \\
A4 (Muğla) & 0.14 & 0.14 & 0.14 & - \\
\hline
\end{tabular}

$\mathrm{C}_{12}=\{\}, \mathrm{C}_{13}=\{\}, \mathrm{C}_{14}=\{1,2\}, \mathrm{C}_{21}=\{1,2,3\}, \mathrm{C}_{23}=$ $\{2,3\}, \mathrm{C}_{24}=\{1,2\}, \mathrm{C}_{31}=\{1,2,3\}, \mathrm{C}_{32}=\{1,2,3\}, \mathrm{C}_{34}=\{1,2\}$, $\mathrm{C}_{41}=\{3\}, \mathrm{C}_{42}=\{3\}, \mathrm{C}_{43}=\{3\}$

\section{Discordance Sets}

In this set, two alternatives are compared and low-value ones are chosen. Table 23 is obtained by applying the necessary procedures.

Table 23: Discordance Index Values.

\begin{tabular}{lllll}
\hline & A1 (Denizli) & A2 (Izmir) & A3 (Manisa) & A4 (Muğla) \\
\hline A1 (Denizli) & - & 1 & 1 & 1 \\
A2 (İzmir) & 0 & - & 0.73 & 0.46 \\
A3 (Manisa) & 0 & 0 & - & 0.72 \\
A4 (Muğla) & 0.12 & 1 & 1 & - \\
\hline
\end{tabular}

$\mathrm{D}_{12}=\{1,2,3\}, \mathrm{D}_{13}=\{1,2,3\}, \mathrm{D}_{14}=\{3\}, \mathrm{D}_{21}=\{\}, \mathrm{D}_{23}$ $=\{1\}, \mathrm{D}_{24}=\{3\}, \mathrm{D}_{31}=\{\}, \mathrm{D}_{32}=\{\}, \mathrm{D}_{34}=\{3\}, \mathrm{D}_{41}=$ $\{1,2\}, D_{42}=\{1,2\}, D_{43}=\{1,2\}$

\section{Step 6 - Superiority Comparison}

The 0.5475 value obtained below is compared with Table 22 and the larger values take the value " 1 " and the smaller values take the value " 0 ". In this way, Table 24 is formed.

Table 24: F Matrix (Concordance Superiority).

\begin{tabular}{lllll}
\hline & A1 (Denizli) & A2 (İzmir) & A3 (Manisa) & A4 (Muğla) \\
\hline A1 (Denizli) & - & 0 & 0 & 1 \\
A2 (İzmir) & 1 & - & 1 & 1 \\
A3 (Manisa) & 1 & 1 & - & 1 \\
A4 (Muğla) & 0 & 0 & 0 & - \\
\hline
\end{tabular}

$$
\begin{aligned}
& \mathrm{C}=(1 /(\mathrm{m} *(\mathrm{~m}-1))) * \sum \mathrm{C}_{\mathrm{kl}} \\
& \mathrm{C}=(1 /(4 *(4-1))) *(0.86+1+0.57+0.86+1+1+0.86+0.14+
\end{aligned}
$$

$0.14+0.14)=0.5475$
The 0.5858 value obtained below is compared with Table 23 and the larger values take the value " 1 " and the smaller values take the value " 0 ". In this way, Table 25 is formed.

Table 25: G Matrix (Discordance Superiority).

\begin{tabular}{lllll}
\hline & A1 (Denizli) & A2 (İzmir) & A3 (Manisa) & A4 (Muğla) \\
\hline A1 (Denizli) & - & 1 & 1 & 1 \\
A2 (İzmir) & 0 & - & 1 & 0 \\
A3 (Manisa) & 0 & 0 & - & 1 \\
A4 (Muğla) & 0 & 1 & 1 & - \\
\hline
\end{tabular}

$$
\begin{aligned}
& \mathrm{D}=(1 /(\mathrm{m} *(\mathrm{~m}-1))) * \sum \mathrm{D}_{\mathrm{kl}} \\
& \mathrm{D}=(1 /(4 *(4-1))) *(1+1+1+0.73+0.46+0.72+0.12+1+1)=
\end{aligned}
$$
0.5858

Step 7 - Calculation of Net Concordance and Discordance Indices

Table 26 and Table 25 values are applied and Table 26 is obtained.

Table 26: Application of Step 7.

\begin{tabular}{lllll}
\hline & A1 (Denizli) & A2 (ízmir) & A3 (Manisa) & A4 (Muğla) \\
\hline A1 (Denizli) & - & 0 & 0 & 1 \\
A2 (İzmir) & 0 & - & 1 & 0 \\
A3 (Manisa) & 0 & 0 & - & 1 \\
A4 (Muğla) & 0 & 0 & 0 & - \\
\hline
\end{tabular}

AHP based ELECTRE I method results are as follows. A4 < A3 $<$ A2 / A4 $<$ A1. According to the results, A2 is the most critical region. For this reason, A2 information is used in goal programming.

\subsection{Application and result for mathematical model}

In this application, the A2 alternative province, which is obtained as the riskiest from AHP based ELECTRE I method, is discussed. The purpose of this case is to form a crisis center in the most suitable region in the riskiest region in case of a natural disaster. The appropriate crisis center location should be in a near and high-populated area that will increase the response rate.

A2 alternative is İzmir province. Districts in İzmir provinces are used as the $\mathrm{j}$ index. There are 30 districts in İzmir province. These districts are Aliağa, Balçova, Bayındır, Bayraklı, Bergama, Beydağ, Bornova, Buca, Çeşme, Çiğli, Dikili, Foça, Gaziemir, Güzelbahçe, Karabağlar, Karaburun, Karşıyaka, Kemalpaşa, Kınık, Kiraz, Konak, Menderes, Menemen, Narlıdere, Odemis, Seferihisar, Selcuk, Tire, Torbali and Urla. $w_{j}$ values are entered into the system as population values (URL 1) and $\mathrm{u}_{\mathrm{jj}}$ values as 
distances between districts (URL 2). Goals are to choose the district where the population is high and the distance to other regions is minimum. In the goal programming result, the most suitable district is obtained as the district of Karaburun. The p-value is 1 .

\section{CONCLUSIONS}

Natural disasters consisting of earthquake, fire, landslide, storm/typhoon, flood, and many more. etc. are serious situations that pose a risk. When a risk analysis is performed in natural disasters for a region under consideration, one of the all-natural disasters is taken into account in general. However, more than one natural disaster can occur in an area and these situations need to be considered together. In this study, the provinces in the Aegean region of Turkey are taken into account. For the provinces in the Aegean region, the revised Fine Kinney method is used for natural disasters. Since the risk values resulting from the Fine Kinney method are near for more than one province, another method is needed. This method is the AHP based ELECTRE I method. In the AHP part of this method, the criteria are weighted. Then the criteria weighted by AHP are used as inputs in the ELECTRE I method. As a result of the ELECTRE I method, the riskiest province is seen as İzmir province. Since it is considered appropriate to establish a crisis center in İzmir province, a mathematical model is suggested for İzmir province. Goal programming is chosen as a mathematical model. In goal programming, determine the district/districts where the population is high and other districts are near. As a result of the study, the most suitable district in the riskiest city is chosen.

The original contribution of this study is the use of the Fine Kinney method with a new approach, the use of more than one method as an input, and a mathematical model. Besides, the most important part of the study is the consideration of many natural disasters that can occur in a wide area. For future studies, to expand the region and use the revised Fine Kinney method in new areas have been recommended.

Peer-review: Externally peer-reviewed.

Conflict of Interest: The author has no conflict of interest to declare.

Grant Support: The author declared that this study has received no financial support.

Hakem Değerlendirmesi: Dış bağımsız.

Çıkar Çatışması: Yazar çıkar çatışması bildirmemiştir.

Finansal Destek: Yazar bu çalışma için finansal destek almadığını beyan etmiştir.

\section{REFERENCES/KAYNAKÇA}

Almeida, A. T. (2005). Multicriteria modelling of repair contract based on utility and ELECTRE I method with dependability and service quality criteria. Annals of Operations Research, 138(1), 113-126.

AFAD (Ministry of Interior Disaster and Emergency Management Presidency), https://tabb-analiz.afad.gov.tr/Default.aspx, Access Date: 25.04.2020

Chen, N., Chen, L., Tang, C., Wu, Z., Chen, A. (2019). Disaster risk evaluation using factor analysis: a case study of Chinese regions. Natural Hazards, 99(1), 321-335.

Dagsuyu, C., Derse, O., Oturakci, M. (2021). Integrated risk prioritization and action selection for cold chain. Environmental Science and Pollution Research, 28(13), 15646-15658.

Dilley, M., Chen, R. S., Deichmann, U., Lerner-Lam, A. L., Arnold, M. (2005). Natural disaster hotspots: a global risk analysis. The World Bank.

Emblemsvåg, J. (2008). On probability in risk analysis of natural disasters. Disaster Prevention and Management: An International Journal.

Eyre, R., De Luca, F., Simini, F. (2020). Social media usage reveals recovery of small businesses after natural hazard events. Nature communications, 11(1), 1-10.

Ersoy, M., Celik, M. Y., Yeşilkaya, L., \& Colak, O. (2019). Combination of Fine-Kinney and GRA methods to solve occupational health and safety problems. Journal of the Faculty of Engineering and Architecture of Gazi University, 34(2), 751-770.

Ganguly, K. K., Guin K. K. (2013). A fuzzy AHP approach for inbound supply risk assessment. Benchmarking: Int. J. 20(1), 129-146.

Gul, M., Guven, B., Guneri, A. F. (2018). A new Fine-Kinney-based risk assessment framework using FAHP-FVIKOR incorporation. Journal of Loss Prevention in the Process Industries, 53, 3-16.

Guidry, V. T., Margolis, L. H. (2005). Unequal respiratory health risk: using GIS to explore hurricane-related flooding of schools in Eastern North Carolina. Environmental research, 98(3), 383-389.

Guo, J., Zhao, S., Huang, C. (2017). Valid historical data for probabilistic risk analysis in natural disasters. Human and Ecological Risk Assessment: An International Journal, 23(3), 474-493.

Hatami-Marbini, A., Tavana, M. (2011). An extension of the Electre I method for group decision-making under a fuzzy environment. Omega, 39(4), 373-386.

Hong, J. D., Jeong, K. Y. (2019). Humanitarian supply chain network design using data envelopment analysis and multi-objective programming models. European Journal of Industrial Engineering, 13(5), 651-680.

Ivčević, A., Mazurek, H., Siame, L., Moussa, A. B., \& Bellier, O. (2019). Indicators in risk management: Are they a user-friendly interface between natural hazards and societal responses? Challenges and opportunities after UN Sendai conference in 2015. International Journal of Disaster Risk Reduction, 101301. 
Kinney, G. F., Wiruth, A. D. (1976). Practical risk analysis for safety management (No. NWC-TP-5865). Naval Weapons Center China Lake Ca.

Kokangül, A., Polat, U., Dağsuyu, C. (2017). A new approximation for risk assessment using the AHP and Fine Kinney methodologies. Safety science, 91, 24-32.

Lee, C. H., Lin, S. H., Kao, C. L., Hong, M. Y., Shih, C. L., Chuang, C. C. (2020). Impact of climate change on disaster events in metropolitan cities-trend of disasters reported by Taiwan national medical response and preparedness system. Environmental research, 183, 109186.

Li, N., Liu, X., Xie, W., Wu, J., Zhang, P. (2013). The return period analysis of natural disasters with statistical modeling of bivariate joint probability distribution. Risk Analysis: An International Journal, 33(1), 134-145.

Luchuan, R. E. N. (1999). Advance in risk analysis for regional natural disasters [J]. Advance in Earth Sciences, 3.

Ma, Y., Xu, W., Qin, L., Zhao, X. (2019). Site selection models in natural disaster shelters: a review. Sustainability, 11(2), 399.

Mabrouki, C., Bentaleb, F., Mousrij, A. (2014). A decision support methodology for risk management within a port terminal. Safety Science, 63, 124-132.

Pang, J., Zhang, G., Chen, G. (2011). ELECTRE I Decision Model of Reliability Design Scheme for Computer Numerical Control Machine. JSW, 6(5), 894-900.
Saaty, T. L. (1980). The analytic hierarchy process, new york: Mcgrew hill. International, Translated to Russian, Portuguesses and Chinese, Revised edition, Paperback (1996, 2000), Pittsburgh: RWS Publications, 9, 19-22.

Sukcharoen, T., Weng, J., Teetat, C. (2016). GIS-based flood risk model evaluated by Fuzzy Analytic Hierarchy Process (FAHP). In Remote Sensing for Agriculture, Ecosystems, and Hydrology XVIII (Vol. 9998, p. 999809). International Society for Optics and Photonics.

Supriyadi, B., Windarto, A. P., Soemartono, T. (2018). Mungad,“Classification of natural disaster prone areas in Indonesia using K-means,". Int. J. Grid Distrib. Comput, 11(8), 87-98.

Osipov, V. I., Rumyantseva, N. A., Eremina, O. N. (2019). Living with risk of natural disasters. Russian Journal of Earth Sciences, 19(6).

URL 1, https://www.nufusu.com/ilceleri/izmir-ilceleri-nufusu, Access Date: 01.05.2020.

URL 2, https://www.google.com/maps, Access Date: 01.05.2020.

Yilmaz, F., Ozcan, M. S. (2019). A Risk Analysis and Ranking Application for Lifting Vehicles Used in Construction Sites with Integrated AHP and Fine-Kinney Approach. Advances in Science and Technology Research Journal, 13(3).

Xu, X., Liang, D., Chen, X., Zhou, Y. (2015). A risk elimination coordination method for large group decision-making in natural disaster emergencies. Human and Ecological Risk Assessment: An International Journal, 21(5), 1314-1325. 